tion systems $s^{3}$ have contributed much to improving the comparability of data from separate studies, but the most effective way of reducing methodological bias is by collaboration between workers using the same research protocol in different countries.

One of the earliest examples of a collaborative study, made shortly after the second world war, was a comparison of serum cholesterol levels in American and Italian men. ${ }^{4}$ The finding that the levels were higher and that they continued to rise with age after 35 years in the more CHD-prone Americans helped to stimulate the subsequent world-wide epidemiological study of blood lipids. Collaborative necropsy studies $^{5}$ have shown coronary atherosclerosis to be more common in countries with higher death rates from CHD and that the severity of atherosclerosis is associated with hypertension and diabetes mellitus. A study of American and British post office and telephone workers ${ }^{6}$ showed that evidence of cardiac ischaemia was more frequent in the Americans, in all smoking categories. Factors associated with the Americans' greater adiposity were thought likely to explain their adverse experience of cardiovascular disease. The Seven Country Study ${ }^{7}$ has indicated that blood pressure and serum cholesterol tend to have higher values in men living in countries where CHD is more common but that other factors related to the incidence of $\mathrm{CHD}$ vary from one country to another. Further analysis of these data ${ }^{8}$ has shown that the Americans have an unexplained excess incidence of clinical CHD over the Europeans which is not due to differences in age, systolic blood pressure, serum cholesterol, smoking habit, or body mass. Recently another comparative study has been reported by two groups investigating men aged 40 selected at random in Edinburgh and Stockholm. ${ }^{9}$ Many risk factors were considered, but the greater number of cigarette smokers and higher levels of systolic blood pressure and fasting serum triglycerides in Edinburgh men were thought to be the most significant findings.

However, in these studies it has not been possible to separate the genetic from the potentially modifiable environmental determinants of risk. Studies in which the characteristics of migrants are compared with those remaining in the country of origin have the advantage that comparisons are between people of the same genetic stock. Blood pressure has been shown to be higher in those who have moved from rural or urban areas ${ }^{10} 11$ (though there is some evidence that this might be explained by differences in weight ${ }^{12}$ ); but migrants from countries with low rates of $\mathrm{CHD}$ who move to high risk countries retain lower blood pressure levels when compared with natives of their host country. ${ }^{1314}$ Other factors, including serum lipids, 1-hour blood sugar, and relative weight, have been shown to differ between men living in Japan and Japanese men in Hawaii and California. ${ }^{15} 16$

Nevertheless, migrants may differ from the populations they leave, for reasons associated with their decision to leave. Cassel ${ }^{17}$ has commented that if migrant studies are to be of real value in disentangling the relative importance of physical and psychosocial factors then the usual cross-sectional study is inadequate. The ideal experiment is one in which migrants are identified before they migrate, are compared with nonmigrants of the same population so that selective factors may be identified, and are followed over time in their new environment. A natural experiment of this design has occurred in the planned migration of the Tokelau Islanders to New Zealand, and its evaluation should lead to a greater understanding of the effects of migration than has previously been possible. ${ }^{18}$

The risk factors examined in international studies have tended to be those that have already been identified elsewhere. Because international studies pose problems of control, com- parability, and cost that far exceed those of more limited ambition, their task should be to provide unique insights into the epidemiology of CHD which cannot be obtained from local or regional comparisons within a country. To this end, the World Health Organisation has been encouraging the use of a common basic protocol for the establishment of ischaemic heart disease registers, ${ }^{1920}$ so that the natural history and management of coronary heart attacks may be compared between countries. WHO is also sponsoring a collaborative trial of preventive measures in four European countries. ${ }^{21} \mathrm{~A}$ preventive trial requires such large numbers to detect statistically significant changes in incidence and mortality that it may be difficult for one country to bear the cost of carrying it out alone. By international co-operation the power of the study can be made sufficient, the consistency of the findings examined, and the effects of modifying the risk factors assessed.

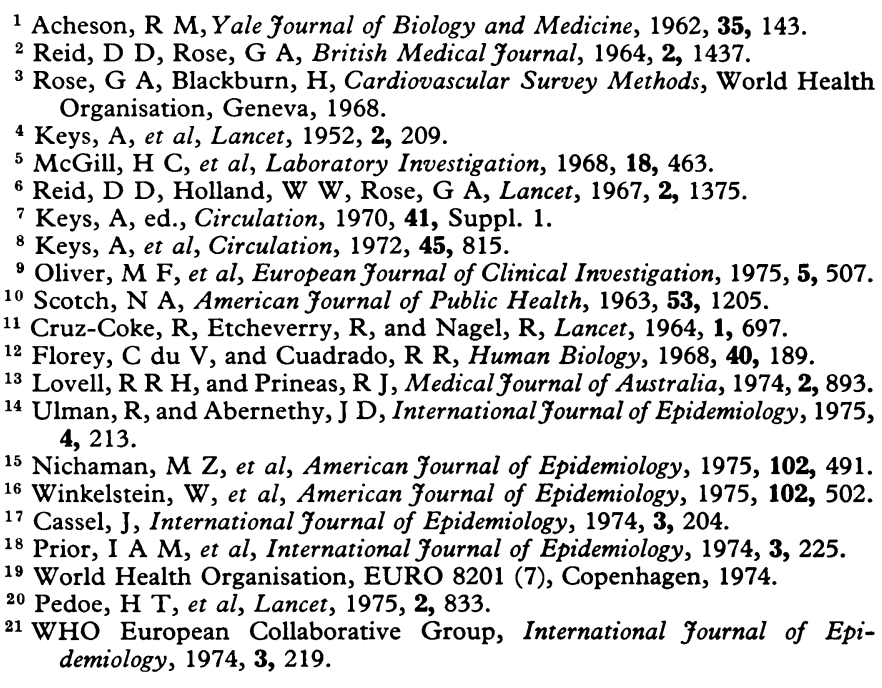

\section{Benign proliferative lesions of the breast}

While clinical attention is rightly focused on carcinoma as the most important disease of the breast, benign mammary proliferations are, in fact, much more frequent than malignant ones. Indeed, the most common lesion of the breast is cystic hyperplasia ${ }^{1}$-also called mammary dysplasia, a term to be preferred since cystic change is not invariable. This condition has its peak incidence in women between the ages of 30 and 50 and is believed to be the result of imbalance of ovarian hormones, probably oestrogen excess and progesterone deficiency. The breast is lumpy, and it may be tender especially during the premenstrual phase of the menstrual cycle. In more advanced cases there are discrete, tense, cystic masses which may fluctuate in size from time to time. The initial histological change is moderate overgrowth and dilatation of ducts, and this may be followed by sufficient epithelial overgrowth to occlude their lumina, a condition called epitheliosis. If there is appreciable ductular proliferation the condition is called adenosis. There is always a moderate proliferation of connective tissue, which may show chronic inflammatory changes. In one variant, sclerosing adenosis, there are masses of tiny ductules surrounded by dense fibrous tissue, and scirrhous carcinoma may be closely simulated. In the final stage there are cysts of varying sizes formed, the largest being the wellknown blue-domed cyst which is lined by thin, atrophic epithelium. 
Another common benign mammary lesion is the fibroadenoma. It occurs predominantly in young women and is seen from time to time in adolescent girls. Clinically it presents as a firm, painless, mobile mass which may, especially in youth, attain giant proportions. Histologically there is a combined proliferation of atypical ducts and connective tissue. The connective-tissue element usually predominates, and it surrounds small ducts in the pericanalicular variety of fibroadenoma, while it invaginates the ducts into elongated, slitlike channels in the intracanalicular variety. The connective tissue is loose, cellular, and sometimes myxomatous in the more florid types of lesions.

A much less common benign tumour is the intraductal papilloma, which occurs most frequently in middle-aged women and presents itself clinically with a serous or bloodstained discharge from the nipple. A small tumour can often be detected in the area of the areola by careful palpation. Histologically there is a proliferation of duct epithelium supported on vascular stalks projecting outwards into a dilated lumen. It is usually a solitary lesion, but occasionally multiple papillomata are present in the smaller ducts.

The relation of these benign lesions to cancer is important. While mammary dysplasia is not precancerous, carcinoma is several times commoner in women with the condition but does not appear to arise especially from the dysplastic tissue. ${ }^{1}$ Fibroadenoma is seldom associated with carcinoma. The rapidly growing giant fibroadenoma closely simulates cancer, but is not malignant. There is a rare condition called cytosarcoma phyllodes, which resembles a giant fibroadenoma clinically and macroscopically and has the histological features of a very exuberant intracanalicular fibroadenoma, but the stroma is hypercellular and shows pleomorphism and mitotic activity sufficient to suggest sarcomatous change. Nevertheless, most of these tumours behave benignly and the invasive and metastasising character of a sarcoma is not present. Nevertheless, several cases do proceed rapidly to sarcomatous change; one in a young girl has recently been reported, ${ }^{2}$ and the condition must be treated with concern. The intraductal papilloma is not precancerous, but the much less common multiple lesion not infrequently proceeds to a special type of intraductal carcinoma. ${ }^{1}$

Though undoubtedly mammary dysplasia is benign, the lesion has the histologically disconcerting tendency to invade normal structures in some cases. Thus perineural and neural invasion has been described on several occasions, especially in sclerosing adenosis, ${ }^{34}$ and recently Eusebi and Azzopardi encountered infiltration of the walls of blood vessels in two cases of sclerosing adenosis. ${ }^{5}$ This discovery prompted them to survey a series of cases of sclerosing adenosis and severe epitheliosis, and they found a $10 \%$ incidence of vascular infiltration. Usually a single vein was affected, but in one case there was infiltration of two veins and an artery. Since infiltration of surrounding structures is accepted as a cardinal histological criterion for diagnosing cancer, evidently great caution is needed before mammary dysplasia is suspected of undergoing malignant change. In this case the cellular and architectural regularity should preclude such an error being made, but the principle remains that diffuse benign epithelial lesions of the breast may show surprising invasive properties.

${ }^{1}$ Haagensen, C D, Diseases of the Breast, 2nd edn. Philadelphia, Saunders, 1971.

2 Hoover, H C, Trestioreanu, A, and Ketcham, A S, Annals of Surgery, $1975,181,279$.

${ }^{3}$ Davies, J D, fournal of Pathology, 1973, 109, 225.

4 Taylor, H B, and Norris, H J, Cancer, 1967, 20, 2245.

5 Eusebi, V, and Azzopardi, J G, Fournal of Pathology, 1976, 118, 9.

\section{Antibiotics again}

One of the paradoxical results of the proliferation of medical journals is that important papers may fail to reach their most appropriate audience. The modern doctor must now not only sample from a general journal for breadth of interest but also read one or more specialist journals for the necessary depth; and a judicious selection has also to be made from books, government and Health Service pamphlets, advertising literature, and medical newspapers.

A recent issue of the New England Fournal of Medicine ${ }^{1}$ carried a possibly overlooked report of the results of a national televised self-assessment test for doctors on the diagnosis and treatment of infectious diseases. The project-the National Antibiotic Therapy Test-attracted a participant audience of 4500 doctors, representing a wide cross-section of professional interests. The test was based on simulated patient problems and was presented to viewers in three parts. A 10-question pretest was followed by a 25-question teaching test in which the clinical illustrations used formed the basis for subsequent teaching by a panel of experts. A final 15-question test was based on the information given during the teaching session. The mean score of $55 \%$ on the pretest improved to $68 \%$ and $71 \%$ on the teaching session and the final test respectively. Only two physicians scored full marks; and one unfortunate recorded only eight correct answers-below par for guessing. An interesting analysis showed the proportion of doctors in different groups achieving a mark of $80 \%$ or better for the complete test. As one might expect, specialists in infectious diseases scored best ( $83 \%$ achieving this target); specialists in internal medicine and paediatrics did less well, $38 \%$ and $24 \%$ respectively meeting the target, but better than family practitioners $(15 \%)$ and surgeons (10\%). Ability to score well deteriorated rather than increased with time since qualification.

High marks might represent only ability to play the game; but the lack of factual knowledge suggested by low marks is likely to be reflected in the realities of patient care. Some of the common difficulties encountered in the test make salutary reading. Only $26 \%$ of doctors knew that penicillin prophylaxis should be started only on the day of dental surgery in a patient at risk to bacterial endocarditis. Only 3\% of doctors were prepared to offer ampicillin but $36 \%$ would offer cloxacillin to patients allergic to penicillin. Other problems included a lack of awareness of drug interactions relating to sulphonamide use, of probable causative organisms in infection after caesarean section, and about the indications for using chloramphenicol. Weakness in knowledge of how to use the laboratory was apparent, and there was clear evidence of lack of knowledge of basic bacteriology- $14 \%$ of doctors identified a Gram-negative bacillus as Streptococcus faecalis or Staphylococcus aureus.

While similar tests of the ability of British specialists and general practitioners would be of interest, anecdotal evidence suggests that many of the problems are common to practice on both sides of the Atlantic Ocean. NHS prescribing statistics from general practice show that antibiotics continue to be used for diarrhoeal illness against the available evidence, that chloramphenicol continues to be prescribed for illnesses other than typhoid fever, and that children and pregnant mothers still receive tetracycline. In addition to wrong use there is the issue of over-use; hospital staff-for some reason exempt from the cost accountability that general practitioners experiencemust accept substantial responsibility for using or recommending inappropriately expensive treatment on many occasions when bacteriological and clinical indications are, at best, borderline. 